\title{
Millisecond Time-Resolved Crystallography using Film Mixing and Plunge Cooling
}

\author{
Matthew Jordan McLeod ${ }^{1}$, Jonathan Clinger $^{1}$, David Moreau ${ }^{1}$, Todd Holyoak ${ }^{2}$, Robert Thorne ${ }^{1}$ \\ ${ }^{1}$ Cornell University, Ithaca NY, United States of America; \\ ${ }^{2}$ University of Waterloo, Waterloo ON, Canada \\ mjm758@cornell.edu
}

\begin{abstract}
Watching biomolecules in motion on biologically relevant time scales has been a long-standing goal of structural biology. Current methodologies allowing for time-resolved crystallographic data collection are mostly through serial methods using microcrystals which are technically challenging experiments with elaborate synchrotron beamline setups, consumption of large amounts of sample, and requiring contributions from many researchers. Here, we propose an alternative methodological setup in order to collect timeresolved data in the millisecond time regime $(>5 \mathrm{~ms})$, suitable for measuring relatively large structural changes that may be ratelimiting in particular cases. Our approach has been to leverage rapid freeze-quenching by robotically plunging our crystals of choice through a substrate film prior to hyperquenching in liquid nitrogen. This method affords many quality of life improvements over current time-resolved methods, such as the potential for a single crystal use per time-point, divorcing the reaction initiation from data collection, and the ability to use the standard mail-in remote data collection available at all synchrotron sources. In order to show proof-of-concept, we used a well characterized metabolic enzyme phosphoenolpyruvate carboxykinase which converts oxaloacetic acid to phosphoenolpyruvate. Our initial experiments uncovered a previously hypothesized state believed to occur directly after phosphoryl transfer and prior to product release. We hope that this method, with its simplicity and ease of access, can allow many structural biology labs to begin time-resolved exploration of suitable systems to uncover further molecular details of enzymes of interest.
\end{abstract}

Keywords: Time-resolved crystallography, Method development, Cryocrystallography 